\title{
Next-Generation Sequencing Identifies Pathogenic Variants in HGF, POU3F4, TECTA, and MYO7A in Consanguineous Pakistani Deaf Families
}

\author{
Xueshuang Mei $\mathbb{D},{ }^{1}$ Yaqi Zhou $\mathbb{D},{ }^{2}$ Muhammad Amjad, ${ }^{3}$ Weiqiang Yang $\mathbb{D},{ }^{1}$ Rufei Zhu, ${ }^{1}$ \\ Muhammad Asif, ${ }^{3}$ Hafiz Muhammad Jafar Hussain, ${ }^{4}$ Tao Yang $\mathbb{D}^{5,6,7}$ Furhan Iqbal ${ }^{\mathbb{D}},{ }^{3}$ \\ and Hongyi Hu ${ }^{1}$ \\ ${ }^{1}$ Department of Otorhinolaryngology, Peking University Shenzhen Hospital, Shenzhen, China \\ ${ }^{2}$ Department of Otorhinolaryngology, Peking University Shenzhen Hospital, Shenzhen Peking University-The Hong Kong University \\ of Science and Technology Medical Center, Shenzhen, China \\ ${ }^{3}$ Institute of Pure and Applied Biology, Bahauddin Zakariya University, Multan, Pakistan \\ ${ }^{4}$ Department of Nephrology, Institute of Nephrology, Ruijin Hospital, Shanghai Jiao Tong University School of Medicine, \\ Shanghai, China \\ ${ }^{5}$ Department of Otorhinolaryngology-Head and Neck Surgery, Ninth People's Hospital, Shanghai Jiao Tong University School \\ of Medicine, Shanghai, China \\ ${ }^{6}$ Ear Institute, Shanghai Jiao Tong University School of Medicine, Shanghai, China \\ ${ }^{7}$ Shanghai Key Laboratory of Translational Medicine on Ear and Nose Diseases, Shanghai, China
}

Correspondence should be addressed to Tao Yang; yangtfxl@sina.com, Furhan Iqbal; furhan.iqbal@bzu.edu.pk, and Hongyi Hu; hyihu@pkuszh.com

Received 2 March 2021; Revised 1 April 2021; Accepted 11 April 2021; Published 22 April 2021

Academic Editor: Renjie Chai

Copyright ( 2021 Xueshuang Mei et al. This is an open access article distributed under the Creative Commons Attribution License, which permits unrestricted use, distribution, and reproduction in any medium, provided the original work is properly cited.

Background. Approximately $70 \%$ of congenital deafness is attributable to genetic causes. Incidence of congenital deafness is known to be higher in families with consanguineous marriage. In this study, we investigated the genetic causes in three consanguineous Pakistani families segregating with prelingual, severe-to-profound deafness. Results. Through targeted next-generation sequencing of 414 genes known to be associated with deafness, homozygous variants c.536del (p. Leu180Serfs $* 20$ ) in TECTA, c.3719 G>A (p. Arg1240Gln) in MYO7A, and c.482+1986_1988del in HGF were identified as the pathogenic causes of enrolled families. Interestingly, in one large consanguineous family, an additional c.706G>A (p. Glu236Lys) variant in the X-linked POU3F4 gene was also identified in multiple affected family members causing deafness. Genotype-phenotype cosegregation was confirmed in all participating family members by Sanger sequencing. Conclusions. Our results showed that the genetic causes of deafness are highly heterogeneous. Even within a single family, the affected members with apparently indistinguishable clinical phenotypes may have different pathogenic variants.

\section{Background}

Congenital hearing loss affects $1 \% 0-2 \%$ of newborns worldwide. Among them, approximately $70 \%$ of deafness is attributable to genetic causes [1]. The genetics of hearing loss is extremely heterogeneous as, to date, more than 120 genes are reported to cause nonsyndromic hearing loss (Hereditary Hearing Loss Homepage; https://hereditaryhearingloss.org).
Similarly, numerous genes are known to cause syndromic hearing loss [2-10]. In recent years, next-generation sequencing (NGS) has been increasingly implemented in the genetic diagnosis of heterogeneous diseases including hearing loss, providing a high-throughput, efficient approach to target hundreds of causative genes or even the whole exome for the identification of pathogenic variants $[11,12]$. Due to the extremely high heterogeneity of genetic hearing 
loss, however, determining the pathogenicity of the candidate variants can be challenging in many cases. For example, rare, benign variants are not always distinguishable from the true pathogenic variants following general guidelines for sequencing data interpretation $[13,14]$. Reporting of rare variants with phenotypic cosegregation in large families, therefore, will provide valuable references for genetic diagnosis of deafness in isolated cases [14].

The deafness-associated genes play diverse roles in the development, function, and maintenance of the inner ear. Variants in these genes correspondently lead to variable auditory phenotypes in regard to onset, severity, progression, audiogram profile, and accompanying syndromic features [1]. In some cases, different variants in the same gene, such as $M Y O 7 A$, may result in distinct phenotypes in nonsyndromic deafness DFNA11 and DFNB2 $[15,16]$ or syndromic deafness USH1B [17]. Documentation and analysis of the genotype-phenotype correlation covering a broad range of novel or previously less characterized variants are therefore necessary to facilitate genetic diagnosis of hearing loss.

Approximately $80 \%$ of genetic deafness are inherited in an autosomal recessive manner [18]. In many cases, they are closely related to consanguineous marriage, which is common in regions of the Middle East, South Asia, South America, and Africa $[19,20]$. Studies in Iranian and Palestinian populations, for example, showed that, respectively, 65\% and $58 \%$ of deaf children were born to parents having consanguineous marriage $[21,22]$. A number of deafnessassociated genes are discovered by linkage analyses based on homozygosity mapping of large consanguineous deaf families $[23,24]$.

In this study, we performed targeted NGS in three Pakistani consanguineous families and identified novel variants in TECTA and POU3F4 and previously reported variants in $M Y O 7 A$ and $H G F$ as the pathogenic causes for prelingual, severe-to-profound deafness. Interestingly, in a large, multigenerational consanguineous family, we identified two separated variants in $H G F$ and POU3F4, illustrating the complex genetic heterogeneity of deafness.

\section{Materials and Methods}

2.1. Subjects and Clinical Evaluations. Three consanguineously married families (PK-DD-KA-01 (Figure 1), PK-DDRP-01 (Figure 2), and PK-DB-OKA-01 (Figure 3)) were enrolled from three districts (Muzafargarh, Rajanpur, and Okara, respectively) in Punjab (Pakistan) with multiple individuals suffering from deafness. Written informed consents were obtained from all participants and/or their parents before their enrollment in this study. Family members affected with deafness were examined in the ear, nose, and throat (ENT) wards of their respective District Head Quarter Hospital by medical specialists. Clinical evaluations included complete medical history interview, comprehensive physical examination, and pure tone audiometry (PTA). Otoscopic examination was performed to exclude hearing loss due to infections, trauma, or other environmental factors. The vestibular function was evaluated by medical history inquiry and behavioral testing. This study was approved by the Research Ethics Committee of the Peking University Shenzhen Hospital.

2.2. Genetic Analysis. Genomic DNA was extracted from peripheral blood samples of the enrolled subjects by using the QIAamp DNA Blood Mini Kit (QIAGEN, Shanghai). Targeted NGS was performed in the proband from each family (arrows in Figures 1(a), 2(a), and 3(a)) plus individual IV-5 from Family PK-DB-OKA-01. The customized capture panel (MyGenostics, Beijing) targeted the exonic region of 414 known deafnessassociated genes; in addition to the exonic region, the panel also included all intronic, intergenic, and noncoding regions containing variants that reported in HGMD (147 genes, 751 variants) (Supplementary Table S1). Candidate pathogenic variants were defined as nonsynonymous (including nonsense, missense, splice-site, and indels) variants with minor allele frequencies (MAFs) less than 0.005 in public databases including 1000 Genomes, dbSNP, and GnomAD. Potential pathogenic effect of the candidate variants was evaluated by in silico tools MutationTaster, PROVEAN, SIFT, and PolyPhen-2. Cosegregation of the deafness phenotype and the pathogenic variants was confirmed in participating family members by PCR amplification and Sanger sequencing; the results were shown in each pedigree map. Pathogenicity of the variants was classified following the guidelines of ACMG 2015 [13].

\section{Results}

3.1. Clinical Characterization. There are at least seven subjects in family PK-DD-KA-01 (Figure 1(a)), 2 in PKDD-RP-01 (Figure 2(a)), and 22 in PK-DB-OKA-01 (Figure 3(a)) that were suffering from bilateral, prelingual, severe-to-profound sensorineural hearing loss (Figures 1(c), 2(c), and 3(c)). All affected subjects in Families PK-DDKA-01 and PK-DD-RP-01 and half (11/22) of the affected subjects in Family PK-DB-OKA-01 were born to parents with consanguineous marriage. No ear malformation, vestibular dysfunction, developmental abnormality, or syndromic symptom were identified in enrolled subjects.

3.2. Identification of the Pathogenic Variants in Probands. Targeted NGS of 414 known deafness genes was performed on probands of the three families. Homozygous variants c.536del (p. Leu180Serfs $* 20$ ) in TECTA (NM_005422.4), c.3719 G>A (p. Arg1240Gln) in MYO7̄A (NM_ 001127180.2), and c.482+1986_1988del in HGF (NM_ 000601.6) were identified as the candidate pathogenic variants in probands IV-4 of Family PK-DD-KA-01 (Figure 1(a)), IV4 of Family PK-DD-RP-01 (Figure 2(a)), and IV-24 of Family PK-DB-OKA-01 (Figure 3(a)), respectively. All candidate variants have minor allele frequencies lower than 0.0001 in the public database gnomAD and are categorized as likely pathogenic based on ACMG guidelines (Table 1).

3.3. Identification of a Second Pathogenic Variant in Family $P K-D B-O K A-01$. Sanger sequencing confirmed that homozygous variants c.536del (p. Leu180Serfs $* 20$ ) in TECTA and c.3719 G>A (p. Arg1240Gln) in MYO7A segregated with the deafness in all participating members in Families PK- 
I

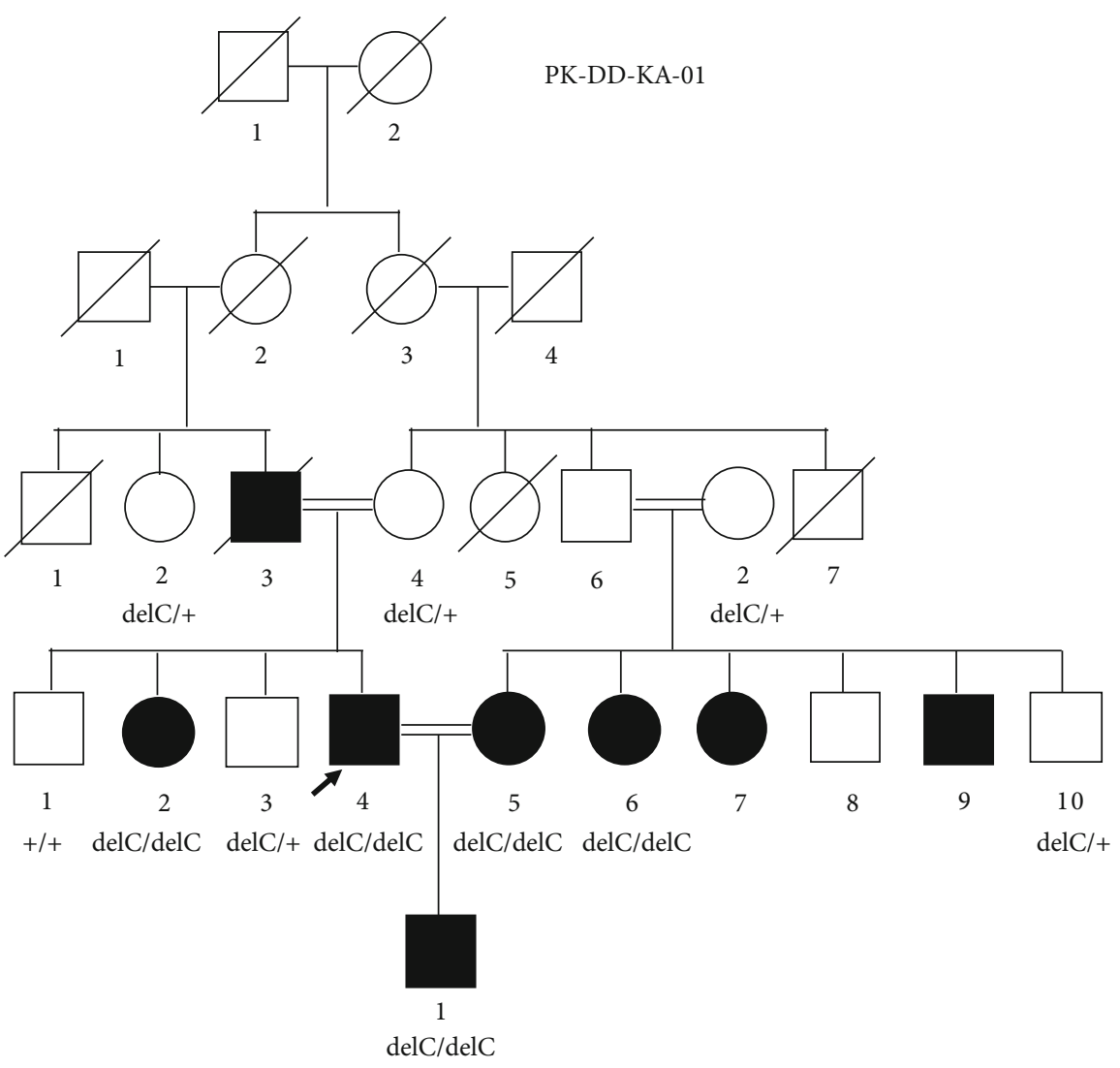

PK-DD-KA-01

II

(a)

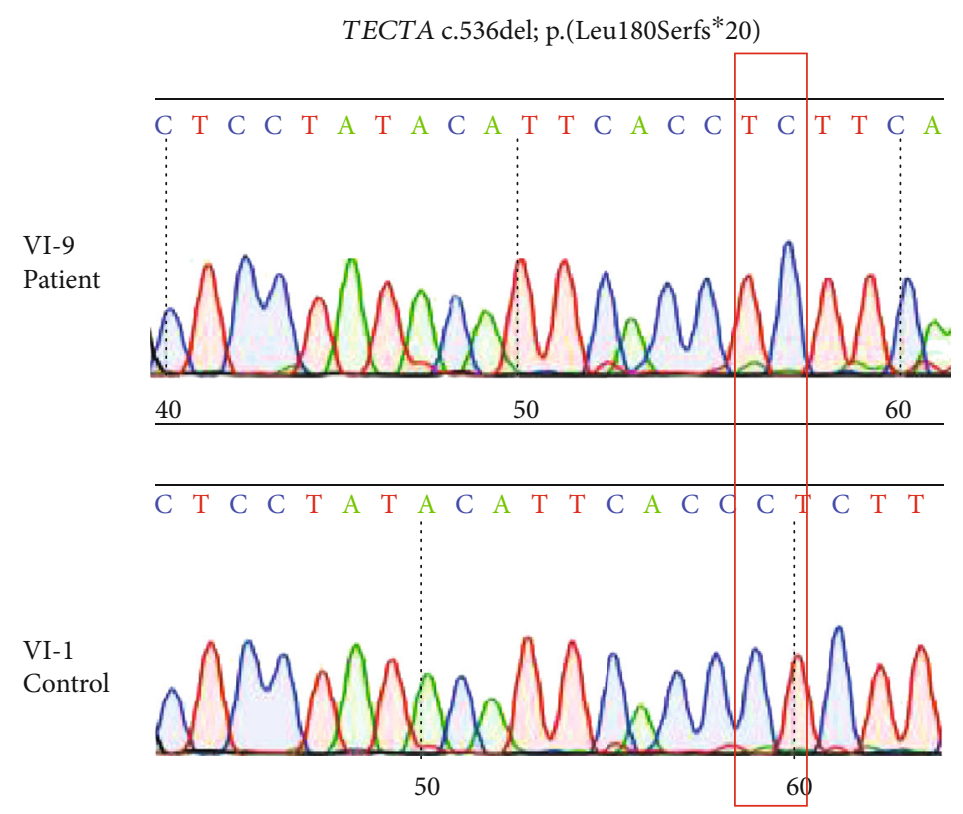

(b)

FIgURE 1: Continued. 


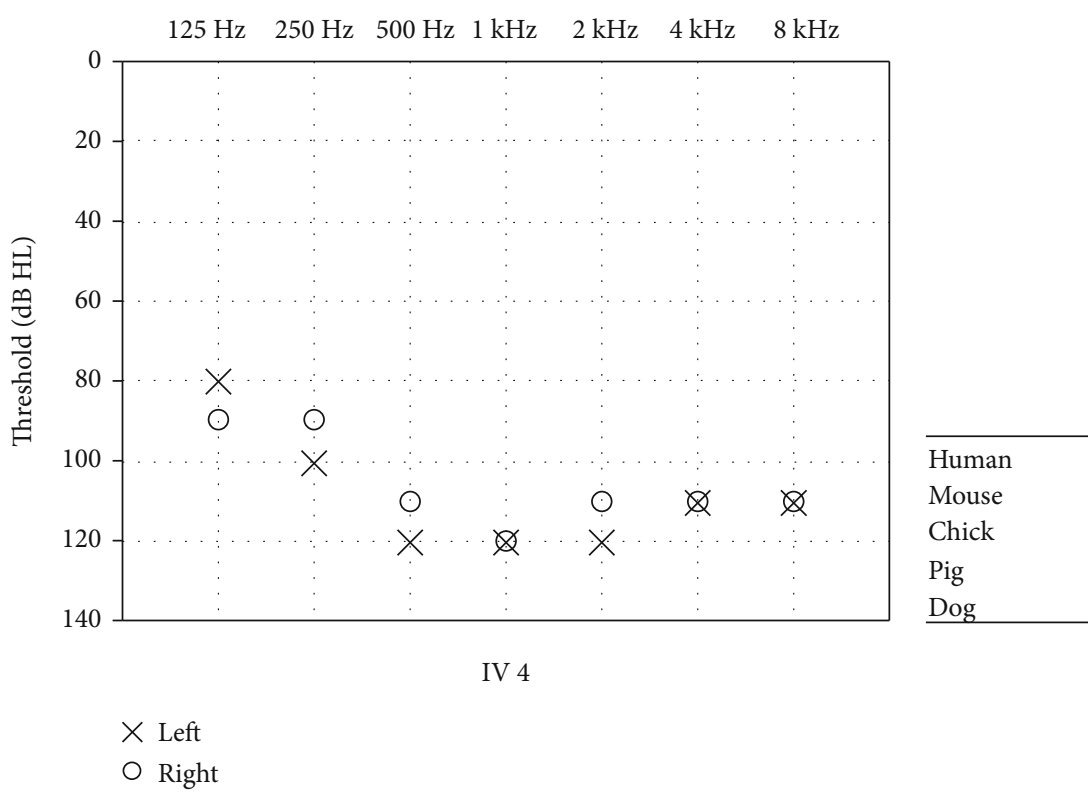

(c)

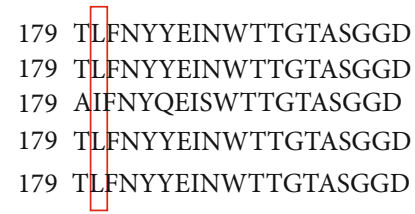

(d)

Figure 1: Genetic and phenotypic characterization of Family PK-DD-KA-01. (a) Pedigree and genotype showing the c.536del (p. Leu180Serfs $* 20$ ) variant in TECTA. (b) Sanger sequencing of the c.536del (p. Leu180Serfs $* 20$ ) variant in affected and unaffected family members. (c) Pure tone audiometry showing the bilateral profound hearing loss in affected family member IV-4. (d) Multiple sequence alignment showing the conservation of the L180 residue in different species.

DD-KA-01 (Figure 1(b)) and PK-DD-RP-01 (Figure 2(b)), respectively. In Family PK-DB-OKA-01, however, five deaf members (IV-4, IV-5, IV-6, III-10, and III-17, marked blue in (Figure 3(a))) were either heterozygous or wild type for variant c.482+1986_1988del in HGF (Figure 3(b)). A second round of targeted NGS on subject IV-5 in this family identified a hemizygous c.706G > A (p. Glu236Lys) variant in the Xchromosome gene POU3F4 (NM_000307.5), a causative gene for X-linked nonsyndromic deafness DFNX2. The c.706G >A (p. Glu236Lys) variant segregated with the deafness in the five aforementioned male family members, but it was not detected in any other family members (Figures 3(a) and 3(b)). This novel variant is not seen in the gnom $\mathrm{AD}$ databases and has not been previously reported in association with hearing loss. It substitutes an evolutionarily conserved, acidic residue glutamic acid to an alkaline residue lysine at position 236 (Figure 3(e)) and is predicted to be deleterious or probably damaging by in silico tools MutationTaster, PROVEAN, SIFT, and PolyPhen-2 (Table 1).

\section{Discussion}

Hearing loss is one of the major disabilities worldwide, which is often induced by loss of sensory hair cells [25-29] and spiral ganglion neurons [30-34] in the inner ear cochlea. Hearing loss could be caused by genetic factors, aging, chronic cochlear infections, infectious diseases, ototoxic drugs, and noise exposure [35-42], and genetic factors account for around $70 \%$ of the hearing loss. In the present study, we are reporting the genetic causes of the prelingual, severe-to-profound deafness in three consanguineous Pakistani families through targeted NGS approach. Variants c.536del (p. Leu180Serfs $* 20$ ) in TECTA and c.706G >A (p. Glu236Lys) in POU3F4 are novel, while c.3719 G>A (p. Arg1240Gln) in MYO7A and c.482 +1986_1988del in HGF were previously reported in only very limited cases associated with deafness [43, 44]. In support of their pathogenicity, all four variants segregated with multiple affected and unaffected family members consistent with the autosomal recessive or X-linked inheritance modes. Considering that homozygosity of rare variants is quite rare in the general population, the data generated during the present study will provide valuable genetic evidence regarding the genetic basis of deafness.

The phenotypes of the three families in this study are all characterized as prelingual, severe-to-profound sensorineural hearing loss. Consistently, a similar type of hearing loss has also been associated with many variants in TECTA, MYO7A, HGF, and POU3F4 in previous reports. TECTA encodes $\alpha$-tectorin, which is one of the main components to comprise the tectorial membrane in the cochlea [45]. Unlike dominant TECTA variants, which are associated with milder hearing loss DFNA8/12 (MIM 601543), the recessive TECTA variants often result in prelingual, severe-toprofound hearing loss (DFNB21, MIM 603629) [46-50]. Like several other recessive, truncating variants in TECTA, the c.536del (p. Leu180Serfs $* 20$ ) variant identified in this study causes a frameshift; this will result either in an abortive protein truncated in exon 4 or in no protein at all due to nonsense-mediated mRNA decay [51] and likely results in loss of function (Figure 4(a)). MYO7A is extensively expressed in the hair cells and plays an important role in maintaining stereocilia differentiation and morphology [52, 53]. The c.3719 G>A (p. Arg1240Gln) variant in MYO7A locates in the highly conserved first MyTH4 domain 
I

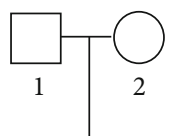

II

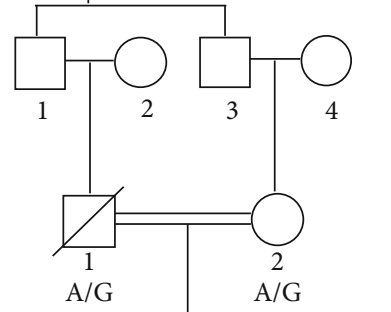

IV

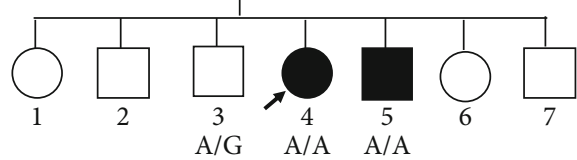

(a)

PK-DD-RP-01
$\mathrm{IV} 4$ Patient

IV2 Control

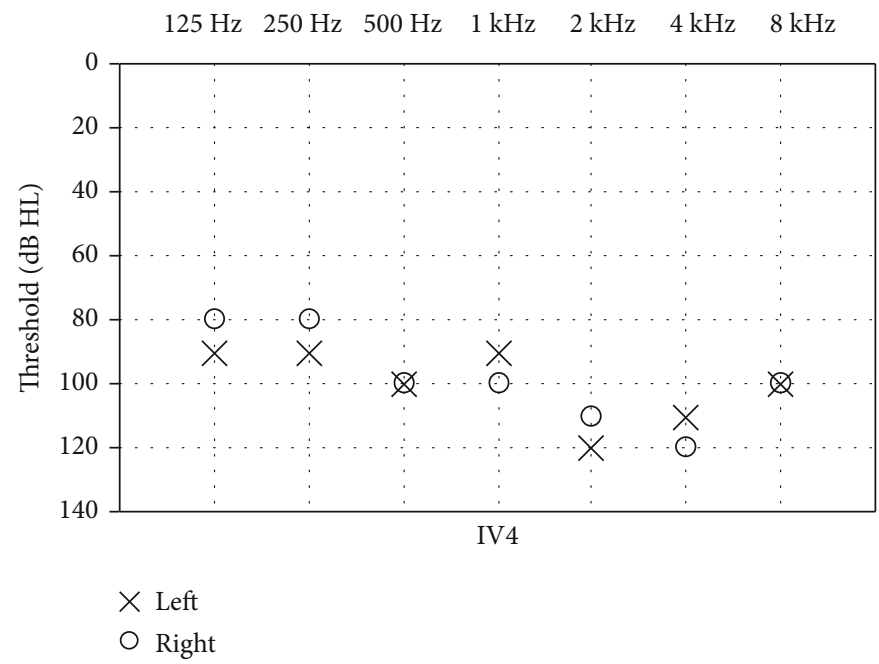

(c)

$\times$ Left
MYO7A c.3719 G>A; p.(Arg1240Gln)

T G G G A C A C A G A C A C A G

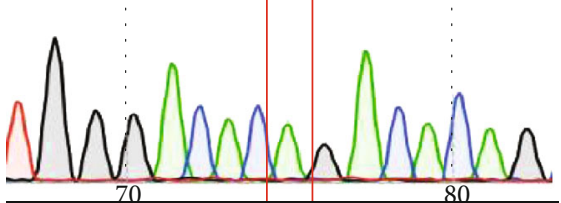

T G G G A C A C G G A C A C A G

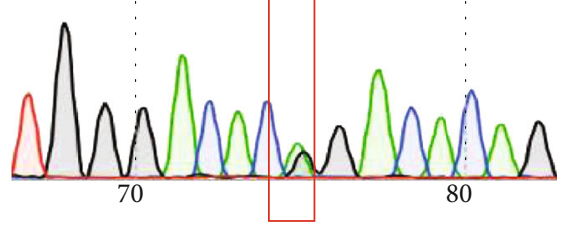

(b)

\begin{tabular}{l}
\hline Human \\
Mouse \\
Dog \\
Zebrafish \\
Sheep \\
\hline
\end{tabular}

TFVNGTRTQPPSWLELQATKSKKPI 1258 TFVNGTRTQPPSWLELQATKSKKPI 1258 TFVNGTRTQPPSWLELQATKSKKPI 1258 TFVNGTRTQPPSWLELQATKSKKPI 1258 TFVNGTRTQPPSWLELQATKSKKPI 1252

(d)

Figure 2: Genetic and phenotypic characterization of Family PK-DD-RP-01. (a) Pedigree and genotype showing the c.3719 G>A (p. Arg1240Gln) variant in MYO7A. (b) Sanger sequencing of the c.3719 G>A (p. Arg1240Gln) variant in affected and unaffected family members. (c) Pure tone audiometry showing the bilateral profound hearing loss in affected family member IV-4. (d) Multiple sequence alignment showing the conservation of the R1240 residue in different species.

(Figure 4(b)). It has been previously reported to be associated with Usher syndrome 1B (MIM 276900), characterized by congenital, severe-to-profound hearing loss, and late-onset retinitis pigmentosa before or after puberty $[44,54]$. Since the two affected children with the c.3719 G>A (p. Arg1240Gln) homozygous variant in our study were 7 and 9 years old without apparent visual abnormality, the potential visual dysfunction remains to be determined at older age. HGF encodes a hepatocyte growth factor and is expressed in the stria vascularis of the mouse inner ear. The c.482+1986_1988del variant identified in this study has been previously reported to cause prelingual, profound deafness (DFNB39, MIM 608265) [43]. A Hgf 10 bp deletion in homozygous mutant mice, which fully encompasses the $3 \mathrm{bp}$ deletion in humans, also displayed profound hearing loss at 4 weeks age (Figure 3(d)) [55]; Hgf 10 bp deletion in homozygous mice causes low expression of $\mathrm{Hg} f$ in the cochlea, which leads to developmental defect of the stria vascularis and reduced endocochlear potential in the cochlea [55]. The $3 \mathrm{bp}$ deletion in the intronic region possibly has a similar mechanism to cause hearing loss. POU3F4 encodes a POU domain transcription factor expressed in a spiral ligament and spiral limbus [56]. Most DFNX2 (MIM 304400) patients with POU3F4 variants have profound hearing loss with or without developmental abnormality of the conductive components $[57,58]$. Although the temporal bone abnormities could not be confirmed in this family, the PTA results of affected males in the family only presented sensorineural hearing loss, which is less common than mixed hearing loss in patients with POU3F4 variants. The novel c.706G>A (p. Glu236Lys) variant identified in this study is located in the highly conserved POU-specific domain (Figure 4(c)), which is similar to a previously reported c.707A $>$ C; p. (Glu236Ala) variant in a Turkey family [59]. Overall, our results are consistent with known genotype-phenotype correlation of the corresponding genes. 


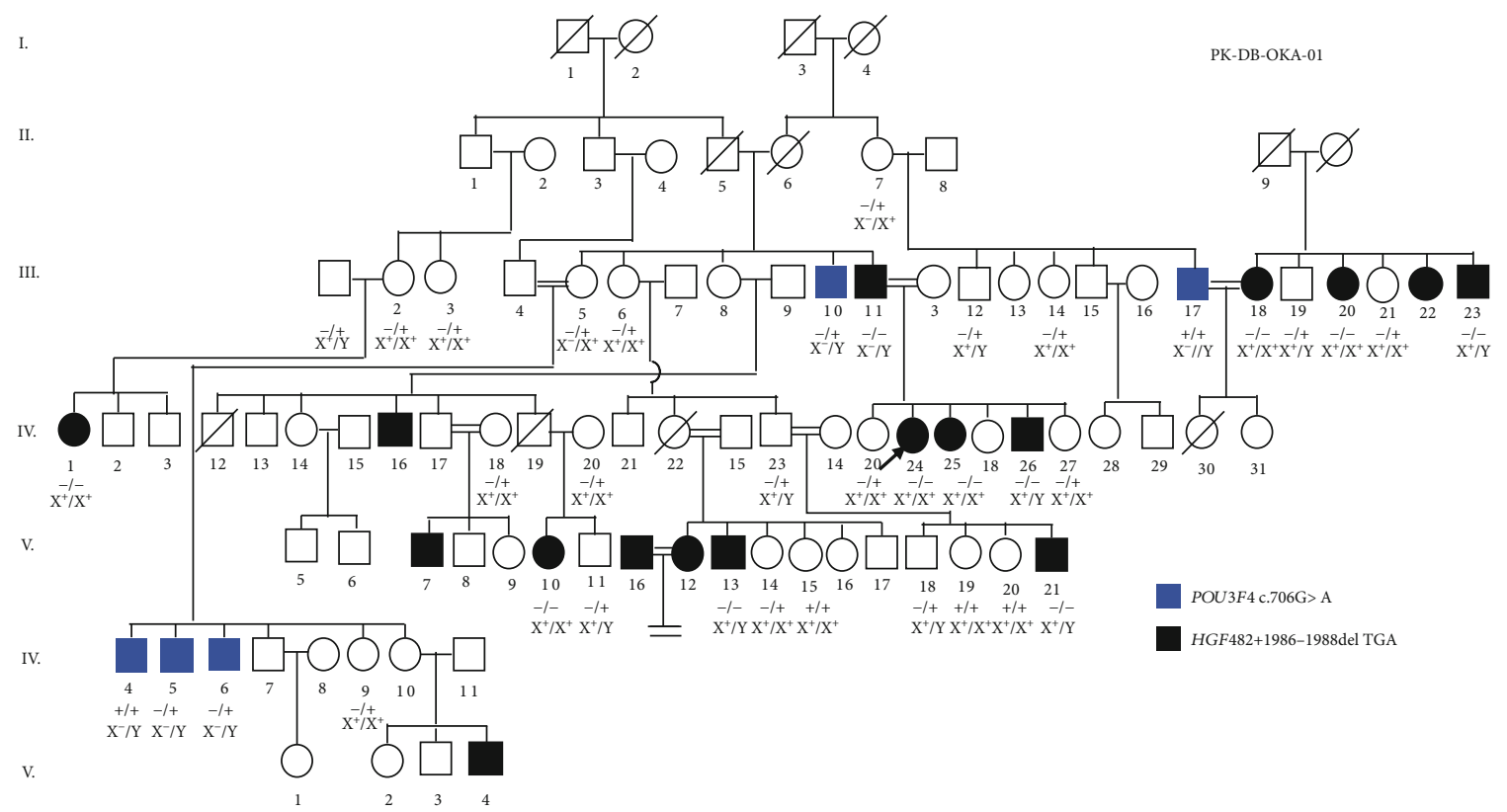

(a)

HGF482+1986-1988del

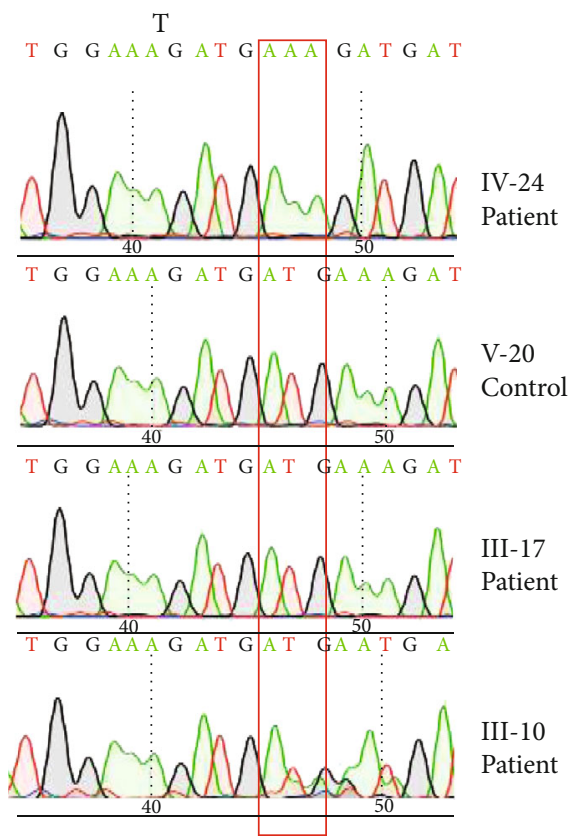

POU $3 F 4$ c. 706G > A;

p.(Glu236Lys [
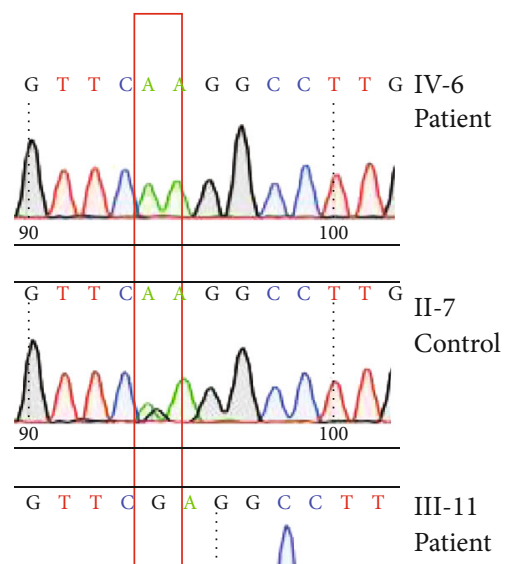

Mandhe

(b)

Figure 3: Continued. 

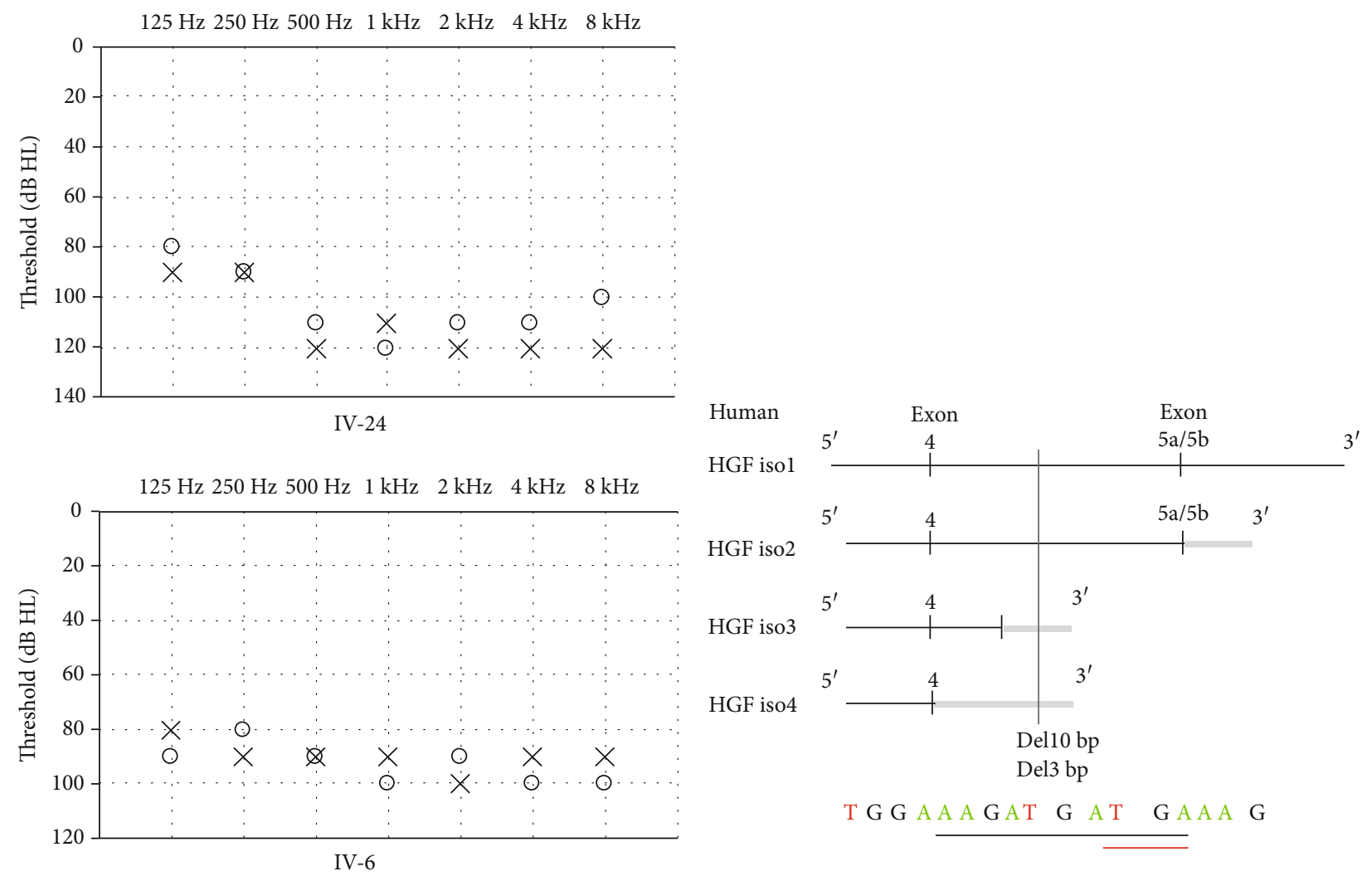

$\times$ Left

$\bigcirc$ Right

(c)

(d)

\begin{tabular}{l}
\hline Human \\
Mouse \\
Dog \\
Pig \\
Sheep \\
\hline
\end{tabular}

TLYGNVFSQTTICRFEG LQL 240 TLYGNVFS QTTICRFEA LQL 240 TLYGNVFSQTTICRFEALQL 240 TLYGNVFSQTTICRFEA LQL 240 TLYGNVFS QTTICRFEA LQL 240

(e)

Figure 3: Genetic and phenotypic characterization of Family PK-DB-OKA-01. (a) Pedigree and genotypes showing the 482+1986-1988del variant in HGF (marked as -) and the c.706G >A (p. Glu236Lys) variant in POU3F4 (marked as $\mathrm{X}^{-}$). (b) Sanger sequencing of the 482 +1986 -1988del and c.706G $>$ A (p. Glu236Lys) variants. (c). Pure tone audiometry showing the bilateral profound hearing loss in affected family members IV-24 and IV-6. (d) Position of the 3 bp deletion in human HGF and the 10 bp deletion in mouse $H g f$ that both lead to profound hearing loss. (e) Multiple sequence alignment showing the conservation of the E236 residue in different species.

As summarized above, in this study, rare pathogenic variants were identified in four separate deafness-associated genes, TECTA, MYO7A, HGF, and POU3F4, which have distinct expression profiles and functions in the inner ear. Nevertheless, all four variants resulted in an almost uniformed type of prelingual, severe-to-profound deafness, representing yet another example of extremely high genetic heterozygosity for hearing loss. Interestingly, within the large pedigree of Family PK-DB-OKA-01 with multiple consanguineous marriages, five of the fifteen affected individuals actually have a hemizygous, $\mathrm{X}$-linked variant as a separate cause of hearing loss irrelevant to the consanguineous marriage pattern (Figure 3(a)). Since most novel deafness-causative genes were originally identified through genetic analysis of such large pedigrees based on assumptions that all affected family members share a single pathogenic variant, our results suggested that caution should remain against such complexed inheritance patterns involving two or multiple genetic causes.

\section{Conclusions}

In summary, our study of three consanguineous families with prelingual, severe-to-profound deafness revealed a rather heterogeneous variant spectrum in the corresponding Pakistani deaf communities. Next-generation sequencing illustrates its advantages in resolving such complexed cases. 


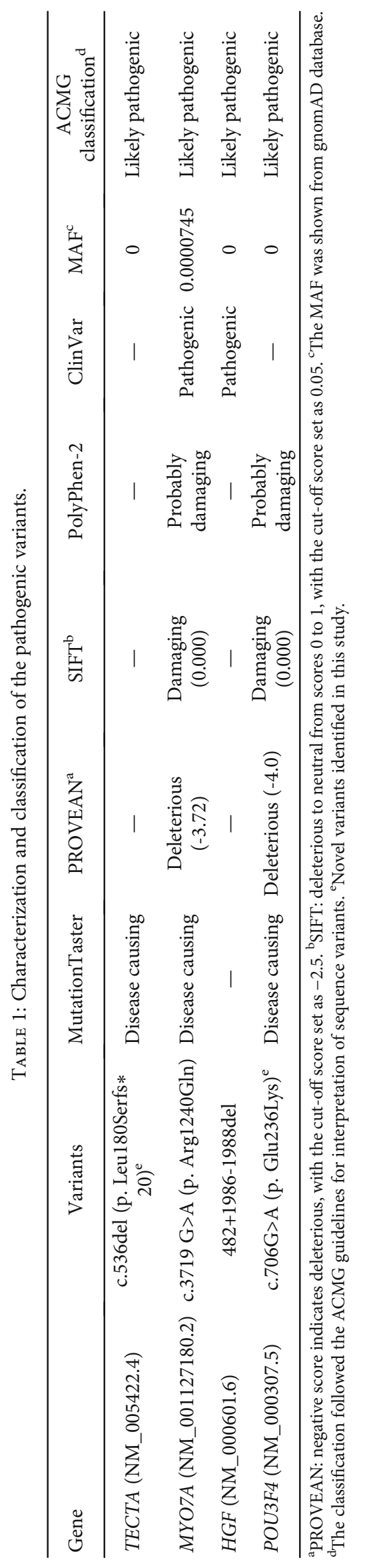




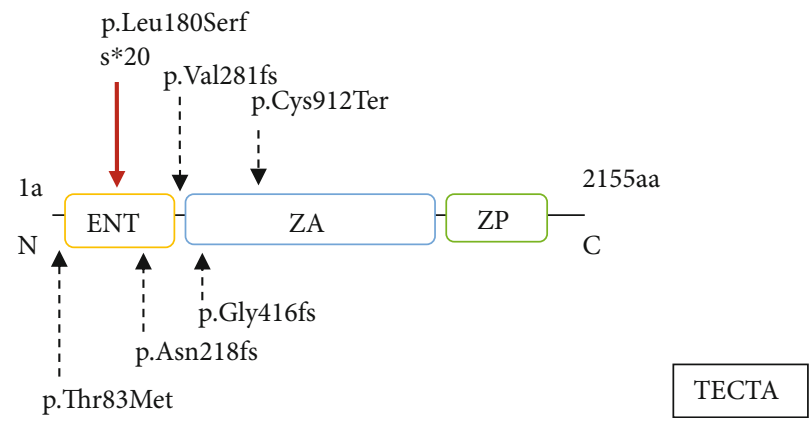

(a)

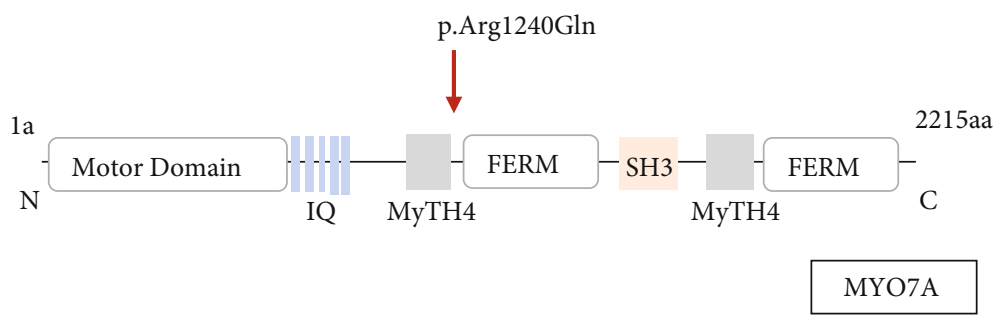

(b)

\begin{tabular}{|c|c|c|}
\hline del $2.6 \mathrm{~kb}, 6.5 \mathrm{~kb}, 7 \mathrm{~kb}, 4.4 \mathrm{~kb}$ & c.249dup(p.Gly84fs) & c. $406 \mathrm{C}>\mathrm{T}\left(\mathrm{p} . \mathrm{G} \ln 136^{*}\right)$ \\
\hline $8 \mathrm{~kb}, 130 \mathrm{~kb}, 20 \mathrm{~kb}, 130 \mathrm{~kb}, 200 \mathrm{~kb}$, & c. 293 C > A (p.Ser98*) & c.410del (p.Pro137fs)c.79C>T(p.Gln27*) \\
\hline $220 \mathrm{~kb}, 530 \mathrm{~kb}, 1200 \mathrm{~kb}$ & c.341G>A (p.Trp114Ter) & c. $478 \mathrm{C}>\mathrm{T}$ (p.Gln160Ter) \\
\hline c.170G >A (p.Trp57Ter) & c.346delG (p.Ala116Profs) & c.499C $>$ T (p.Arg167Ter) \\
\hline c. $232 \mathrm{C}>\mathrm{T}$ (p.Gln78Ter) & c.383delG (p.Gly128 fs) & c. $530 \mathrm{C}>\mathrm{A}\left(\mathrm{p} . \operatorname{Ser} 177^{*}\right)$ \\
\hline c. $235 \mathrm{C}>\mathrm{T}$ (p.Gln79Ter) & c. $385 \mathrm{C}>\mathrm{T}$ (p.Gln129Ter) & c.559G $>$ T(p.Glu187*) \\
\hline
\end{tabular}

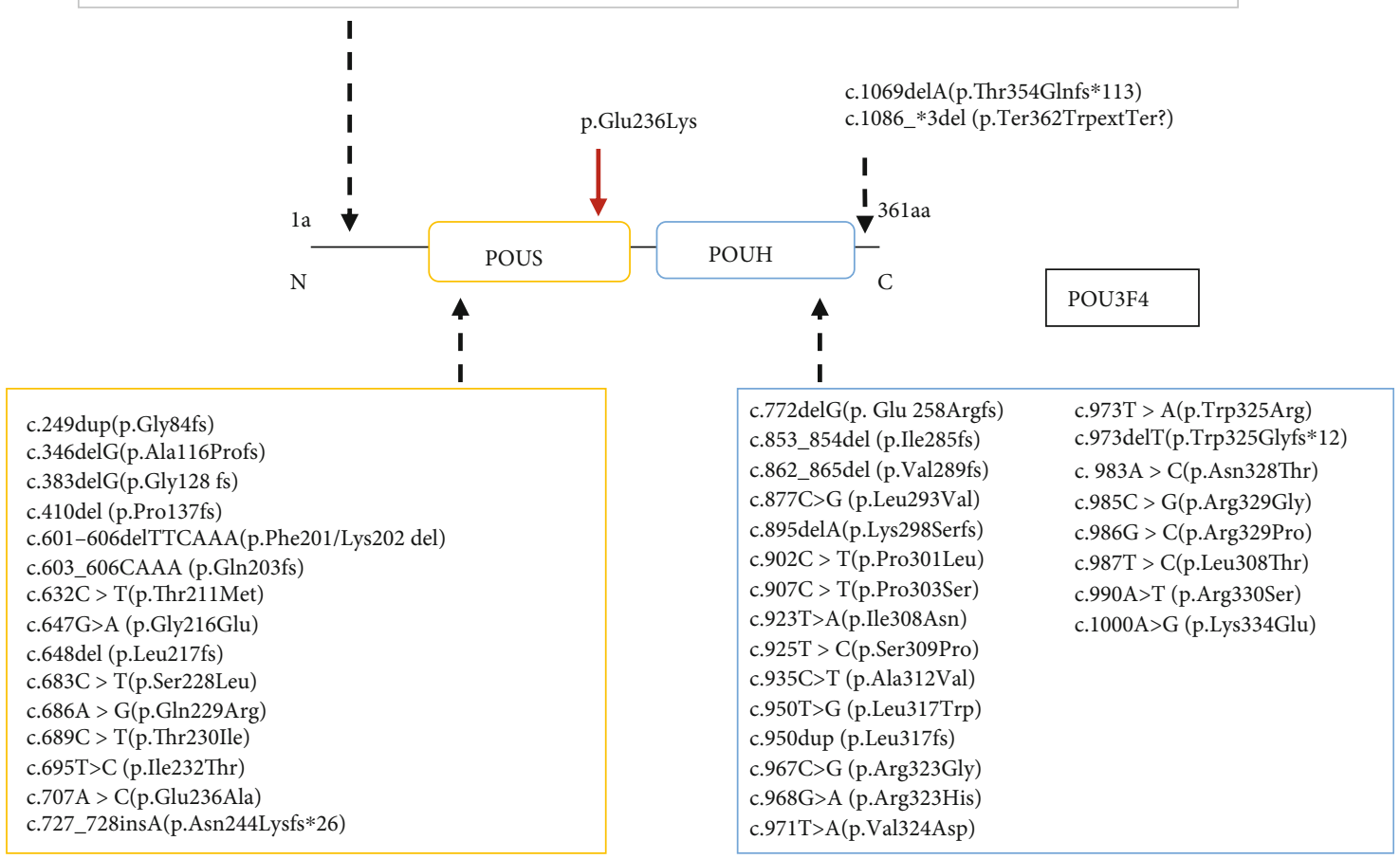

(c)

Figure 4: Domain structure and variant position of TECTA, MYO7A, and POU3F4 proteins. (a) $\alpha$-Tectorin consists of entactin domain $(\mathrm{ENT})$, zonadhesin (ZA) domain, and C-terminal zona pellucida (ZP) domain. Previously reported autosomal recessive variants in TECTA are presented, with the c.536del (p. Leu180Serfs*20) variant highlighted by the red arrow. (b) Myosin VIIA consists of a motor domain, five IQ motif repeats, two large repeats of MyTH4 and FERM domains, and an SH3 domain. The c.3719 G>A (p. Arg1240Gln) variant is highlighted by the red arrow. (c) POU3F4 consists of a POU-specific domain (POUS) and a POU homeodomain (POUH). Previously reported POU3F4 variants are presented, with the c.706G $>$ A (p. Glu236Lys) variant highlighted by the red arrow. 


\section{Data Availability}

The original data is available upon request by contacting the corresponding author.

\section{Conflicts of Interest}

The authors declare no conflict of interest.

\section{Authors' Contributions}

XM, YZ, Muhammad Amjad, TY, FI, and HH contributed to the conceptualization. XM, YZ, WY, RZ, Muhammad Asif, and $\mathrm{HMJH}$ contributed to the methodology. $\mathrm{HH}, \mathrm{YZ}$, and TY contributed to the funding acquisition. XM and YZ wrote the original draft. HH, TY, and FI contributed to the review and editing. All authors read and approved the final manuscript. Xueshuang Mei, Yaqi Zhou, and Muhammad Amjad contributed equally to this work.

\section{Acknowledgments}

This research was supported by the Shenzhen Sanming Project (SZSM201612076 to HH), the China Postdoctoral Science Foundation (2020M672757 to YZ), and the Shanghai Municipal Education Commission-Gaofeng Clinical Medicine Grant (20152519 to TY).

\section{Supplementary Materials}

Table S1: 414 genes associated with deafness that were sequenced by targeted NGS. (Supplementary Materials)

\section{References}

[1] C. C. Morton and W. E. Nance, "Newborn hearing screeninga silent revolution," The New England Journal of Medicine, vol. 354, no. 20, pp. 2151-2164, 2006.

[2] A. A. Dror and K. B. Avraham, "Hearing impairment: a panoply of genes and functions," Neuron, vol. 68, no. 2, pp. 293308, 2010.

[3] I. Schrijver, "Hereditary non-syndromic sensorineural hearing loss: transforming silence to sound," The Journal of Molecular Diagnostics: JMD, vol. 6, no. 4, pp. 275-284, 2004.

[4] Y. He, X. Lu, F. Qian, D. Liu, R. Chai, and H. Li, "Insmla is required for zebrafish posterior lateral line development," Frontiers in Molecular Neuroscience, vol. 10, no. 241, 2017.

[5] Y. Wang, J. Li, X. Yao et al., "Loss of CIB2 causes profound hearing loss and abolishes mechanoelectrical transduction in mice," Frontiers in Molecular Neuroscience, vol. 10, no. 401, 2017.

[6] C. Zhu, C. Cheng, Y. Wang et al., "Loss of ARHGEF6 causes hair cell stereocilia deficits and hearing loss in mice," Frontiers in Molecular Neuroscience, vol. 11, p. 362, 2018.

[7] Q. Fang, Y. Zhang, P. da et al., "Deletion of Limk1 and Limk2 in mice does not alter cochlear development or auditory function," Scientific Reports, vol. 9, no. 1, p. 3357, 2019.

[8] Z. He, Q. Fang, H. Li et al., "The role of FOXG1 in the postnatal development and survival of mouse cochlear hair cells," Neuropharmacology, vol. 144, pp. 43-57, 2019.
[9] F. Qian, X. Wang, Z. Yin et al., "The slc4a2b gene is required for hair cell development in zebrafish," Aging, vol. 12, no. 19, pp. 18804-18821, 2020.

[10] J. Lv, X. Fu, Y. Li et al., "Deletion of Kcnj16 in mice does not alter auditory function," Frontiers in Cell and Developmental Biology, vol. 9, no. 250, 2021.

[11] T. Yang, X. Wei, Y. Chai, L. Li, and H. Wu, "Genetic etiology study of the non-syndromic deafness in Chinese Hans by targeted next-generation sequencing," Orphanet Journal of Rare Diseases, vol. 8, no. 1, p. 85, 2013.

[12] S. Zou, X. Mei, W. Yang, R. Zhu, T. Yang, and H. Hu, "Wholeexome sequencing identifies rare pathogenic and candidate variants in sporadic Chinese Han deaf patients," Clinical Genetics, vol. 97, no. 2, pp. 352-356, 2020.

[13] on behalf of the ACMG Laboratory Quality Assurance Committee, S. Richards, N. Aziz et al., "Standards and guidelines for the interpretation of sequence variants: a joint consensus recommendation of the American College of Medical Genetics and Genomics and the Association for Molecular Pathology," Genetics in Medicine: official journal of the American College of Medical Genetics., vol. 17, no. 5, pp. 405-423, 2015.

[14] L. He, X. Pang, H. Liu, Y. Chai, H. Wu, and T. Yang, "Targeted next-generation sequencing and parental genotyping in sporadic Chinese Han deaf patients," Clinical Genetics, vol. 93, no. 4, pp. 899-904, 2018.

[15] S. Riazuddin, S. Nazli, Z. M. Ahmed et al., "Mutation spectrum of MYO7A and evaluation of a novel nonsyndromic deafness DFNB2 allele with residual function," Human Mutation, vol. 29, no. 4, pp. 502-511, 2008.

[16] Y. Kaneko, A. Nakano, Y. Arimoto, K. Nara, H. Mutai, and T. Matsunaga, "The first sporadic case of DFNA11 identified by next-generation sequencing," International Journal of Pediatric Otorhinolaryngology, vol. 100, pp. 183-186, 2017.

[17] G. Lévy, F. Levi-Acobas, S. Blanchard et al., "Myosin VIIA gene: heterogeneity of the mutations responsible for Usher syndrome type IB," Human Molecular Genetics, vol. 6, no. 1, pp. 111-116, 1997.

[18] N. E. Morton, "Genetic epidemiology of hearing impairment," Annals of the New York Academy of Sciences, vol. 630, no. 1 Genetics of H, pp. 16-31, 1991.

[19] M. Fathzadeh, M. A. Babaie Bigi, M. Bazrgar, M. Yavarian, H. R. Tabatabaee, and S. M. Akrami, "Genetic counseling in southern Iran: consanguinity and reason for referral," Journal of Genetic Counseling, vol. 17, no. 5, pp. 472-479, 2008.

[20] D. Alnaqeb, H. Hamamy, A. M. Youssef, and K. Al-Rubeaan, "Assessment of knowledge, attitude and practice towards consanguineous marriages among a cohort of multiethnic health care providers in Saudi Arabia," Journal of Biosocial Science, vol. 50, no. 1, pp. 1-18, 2018.

[21] M. Ajallouyan, S. Radfar, S. Nouhi et al., "Consanguinity among parents of Iranian deaf children," Iranian Red Crescent Medical Journal, vol. 18, no. 11, p. e22038, 2016.

[22] A. Abu Rayyan, L. Kamal, S. Casadei et al., "Genomic analysis of inherited hearing loss in the Palestinian population," Proceedings of the National Academy of Sciences of the United States of America, vol. 117, no. 33, pp. 2007020076, 2020.

[23] R. W. Collin, E. Kalay, J. Oostrik et al., "Involvement of DFNB59 mutations in autosomal recessive nonsyndromic hearing impairment," Human Mutation, vol. 28, no. 7, pp. 718-723, 2007. 
[24] S. Y. Khan, S. Riazuddin, M. Tariq et al., "Autosomal recessive nonsyndromic deafness locus DFNB63 at chromosome 11q13.2-q13.3," Human Genetics, vol. 120, no. 6, pp. 789$793,2007$.

[25] J. Qi, L. Zhang, F. Tan et al., "Espin distribution as revealed by super-resolution microscopy of stereocilia," American Journal of Translational Research, vol. 12, no. 1, pp. 130-141, 2020.

[26] H. Zhou, X. Qian, N. Xu et al., "Disruption of Atg7-dependent autophagy causes electromotility disturbances, outer hair cell loss, and deafness in mice," Cell Death \& Disease, vol. 11, no. 10, p. 913, 2020.

[27] Y. Liu, J. Qi, X. Chen et al., "Critical role of spectrin in hearing development and deafness," Science Advances, vol. 5, no. 4, article eaav7803, 2019.

[28] J. Qi, Y. Liu, C. Chu et al., “A cytoskeleton structure revealed by super-resolution fluorescence imaging in inner ear hair cells," Cell Discovery, vol. 5, no. 1, p. 12, 2019.

[29] Z. H. He, S. Y. Zou, M. Li et al., "The nuclear transcription factor FoxG1 affects the sensitivity of mimetic aging hair cells to inflammation by regulating autophagy pathways," Redox Biology, vol. 28, p. 101364, 2020.

[30] R. Guo, M. Xiao, W. Zhao et al., "2D Ti3C2TxMXene couples electrical stimulation to promote proliferation and neural differentiation of neural stem cells," Acta Biomaterialia, 2020.

[31] R. Guo, S. Zhang, M. Xiao et al., "Accelerating bioelectric functional development of neural stem cells by graphene coupling: implications for neural interfacing with conductive materials," Biomaterials, vol. 106, pp. 193-204, 2016.

[32] R. Guo, J. Li, C. Chen et al., "Biomimetic 3D bacterial cellulosegraphene foam hybrid scaffold regulates neural stem cell proliferation and differentiation," Colloids and surfaces B, Biointerfaces, vol. 200, article 111590, 2021.

[33] R. Guo, X. Ma, M. Liao et al., "Development and application of cochlear implant-based electric-acoustic stimulation of spiral ganglion neurons," ACS Biomaterials Science \& Engineering, vol. 5, no. 12, pp. 6735-6741, 2019.

[34] W. Liu, X. Xu, Z. Fan et al., "Wnt signaling activates TP53induced glycolysis and apoptosis regulator and protects against cisplatin-induced spiral ganglion neuron damage in the mouse cochlea," Antioxidants \& Redox Signaling, vol. 30, no. 11, pp. 1389-1410, 2019.

[35] Z. He, L. Guo, Y. Shu et al., "Autophagy protects auditory hair cells against neomycin-induced damage," Autophagy, vol. 13, no. 11, pp. 1884-1904, 2017.

[36] C. Cheng, Y. Wang, L. Guo et al., "Age-related transcriptome changes in Sox $2+$ supporting cells in the mouse cochlea," Stem Cell Research \& Therapy, vol. 10, no. 1, p. 365, 2019.

[37] F. Tan, C. Chu, J. Qi et al., "AAV-ie enables safe and efficient gene transfer to inner ear cells," Nature Communications, vol. 10, no. 1, p. 3733, 2019.

[38] Y. Zhang, W. Li, Z. He et al., "Pre-treatment with fasudil prevents neomycin-induced hair cell damage by reducing the accumulation of reactive oxygen species," Frontiers in Molecular Neuroscience, vol. 12, p. 264, 2019.

[39] S. Gao, C. Cheng, M. Wang et al., "Blebbistatin inhibits neomycin-induced apoptosis in hair cell-like HEI-OC-1 cells and in cochlear hair cells," Frontiers in Cellular Neuroscience, vol. 13, no. 590, 2020.

[40] S. Han, Y. Xu, J. Sun et al., "Isolation and analysis of extracellular vesicles in a Morpho butterfly wing- integrated microvor- tex biochip," Biosensors \& Bioelectronics, vol. 154, p. 112073, 2020.

[41] S. Zhang, Y. Zhang, Y. Dong et al., "Knockdown of Foxg1 in supporting cells increases the trans-differentiation of supporting cells into hair cells in the neonatal mouse cochlea," Cellular and Molecular Life Sciences: CMLS, vol. 77, no. 7, pp. 14011419, 2020.

[42] Z. Zhong, X. Fu, H. Li et al., "Citicoline protects auditory hair cells against neomycin-induced damage," Frontiers in Cell and Developmental Biology, vol. 8, p. 712, 2020.

[43] J. M. Schultz, S. N. Khan, Z. M. Ahmed et al., "Noncoding mutations of HGF are associated with nonsyndromic hearing loss, DFNB39," American Journal of Human Genetics, vol. 85, no. 1, pp. 25-39, 2009.

[44] T. Jaijo, E. Aller, M. Beneyto et al., "MYO7A mutation screening in Usher syndrome type I patients from diverse origins," Journal of Medical Genetics, vol. 44, no. 3, article e71, 2007.

[45] D. K. Kim, J. A. Kim, J. Park, A. Niazi, A. Almishaal, and S. Park, "The release of surface-anchored $\alpha$-tectorin, an apical extracellular matrix protein, mediates tectorial membrane organization," Science Advances, vol. 5, no. 11, article eaay6300, 2019.

[46] S. Asgharzade, M. A. Tabatabaiefar, M. H. Modarressi et al., "A novel TECTA mutation causes ARNSHL," International Journal of Pediatric Otorhinolaryngology, vol. 92, pp. 88-93, 2017.

[47] O. Diaz-Horta, D. Duman, J. Foster 2nd et al., "Whole-exome sequencing efficiently detects rare mutations in autosomal recessive nonsyndromic hearing loss," PLoS One, vol. 7, no. 11, article e50628, 2012.

[48] S. Naz, F. Alasti, A. Mowjoodi et al., "Distinctive audiometric profile associated with DFNB21 alleles of TECTA," Journal of Medical Genetics, vol. 40, no. 5, pp. 360-363, 2003.

[49] F. Alasti, M. H. Sanati, A. H. Behrouzifard et al., "A novel TECTA mutation confirms the recognizable phenotype among autosomal recessive hearing impairment families," International Journal of Pediatric Otorhinolaryngology, vol. 72, no. 2, pp. 249-255, 2008.

[50] A. Behlouli, C. Bonnet, S. Abdi et al., "A novel biallelic splice site mutation of TECTA causes moderate to severe hearing impairment in an Algerian family," International Journal of Pediatric Otorhinolaryngology, vol. 87, pp. 28-33, 2016.

[51] K. E. Baker and R. Parker, "Nonsense-mediated mRNA decay: terminating erroneous gene expression," Current Opinion in Cell Biology, vol. 16, no. 3, pp. 293-299, 2004.

[52] T. Self, T. Sobe, N. G. Copeland, N. A. Jenkins, K. B. Avraham, and K. P. Steel, "Role of myosin VI in the differentiation of cochlear hair cells," Developmental Biology, vol. 214, no. 2, pp. 331-341, 1999.

[53] S. Li, A. Mecca, J. Kim et al., "Myosin-VIIa is expressed in multiple isoforms and essential for tensioning the hair cell mechanotransduction complex," Nature Communications, vol. 11, no. 1, p. 2066, 2020.

[54] S. G. Jacobson, A. V. Cideciyan, T. S. Aleman et al., "Usher syndromes due to MYO7A, PCDH15, USH2A or GPR98 mutations share retinal disease mechanism," Human Molecular Genetics, vol. 17, no. 15, pp. 2405-2415, 2008.

[55] R. J. Morell, R. Olszewski, R. Tona et al., "Noncoding microdeletion in mouse Hgf disrupts neural crest migration into the stria vascularis, reduces the endocochlear potential, and suggests the neuropathology for human nonsyndromic deafness DFNB39," The Journal of Neuroscience: the official journal of 
the Society for Neuroscience, vol. 40, no. 15, pp. 2976-2992, 2020.

[56] T. Parzefall, S. Shivatzki, D. R. Lenz et al., "Cytoplasmic mislocalization of POU3F4 due to novel mutations leads to deafness in humans and mice," Human Mutation, vol. 34, no. 8, pp. 1102-1110, 2013.

[57] Y. Su, X. Gao, S. S. Huang et al., "Clinical and molecular characterization of POU3F4 mutations in multiple DFNX2 Chinese families," BMC Medical Genetics, vol. 19, no. 1, p. $157,2018$.

[58] M. Bitner-Glindzicz, P. Turnpenny, P. Höglund et al., "Further mutations in brain 4 (POU3F4) clarify the phenotype in the Xlinked deafness, DFN3," Human Molecular Genetics, vol. 4, no. 8, pp. 1467-1469, 1995.

[59] G. Bademci, A. Lasisi, K. O. Yariz et al., "Novel domainspecific POU3F4 mutations are associated with X-linked deafness: examples from different populations," BMC Medical Genetics, vol. 16, no. 1, p. 9, 2015. 